\title{
Advantages and Applications of Cryopreservation in Fisheries Science
}

\author{
S. Tsai ${ }^{1}$ and C. Lin $^{2,3^{*}}$ \\ ${ }^{1}$ Mingdao University, 369; Wen-Hua Road; Peetow; ChangHua 52345; Taiwan. ${ }^{2}$ National Museum of Marine \\ Biology and Aquarium, 2; Houwan Road; Checheng; Pingtung, 944; Taiwan. ${ }^{3}$ National Dong Hwa University, 2 ; \\ Houwan Road; Checheng, Pingtung, 944; Taiwan
}

\begin{abstract}
Cryopreservation is a long-term storage technique to preserve the biological material without deterioration for extended period of time at least several thousands of years. The ability to preserve and store both maternal and paternal gametes provides a reliable source of fish genetic material for scientific and aquaculture purposes as well as for conservation of biodiversity. Successful cryopreservation of fish sperm have been achieved for more than 200 fish species and many fish species have been adequated for the purpose of cryobanking. Cryopreservation of fish embryo is not viable, mainly because of the same limitations as in fish oocytes, i.e., high chilling sensitivity and low membrane permeability. However, cryopreservation of isolated embryonic cells is another option for preserving both maternal and paternal genome. In this paper, an overview of the current state of aquatic species is followed by a discussion on the sperm, embryos, oocytes and embryonic cells - blastomeres.
\end{abstract}

Key words: cryopreservation, sperm, embryo, oocyte, blastomere

\section{INTRODUCTION}

Cryopreservation is a long-term storage technique with very low temperatures to preserve the structurally intact living cells and tissues for extended period of time at a relatively low cost. Cryopreservation is to preserve and store the viable biological samples in a frozen state over extended periods of time. A very important part research in cryopreservation is to reveal the underlying physical and biological responses of the cell and cause of cryoinjury, especially those associated with the phase change of water in extracellular and intracellular environments (Mazur 1984). From the original slow-cooling study, another cryopreservation approach has moved to easier and more efficient technique- vitrification, Cryoprotective agents has to gain access to all the parts of the system. Cryopreservation considers the effects of freezing and thawing. Therefore, the diffusion and osmosis processes have important effects during the introduction of cryoprotective agents, the addition or removal of cryoprotectants, the cooling process, and during thawing. These phenomena are amenable to the experimental design and analysis. Thus, reliable methods can be developed for preserving a very wide range of cells and some tissues. These methods have found widespread applications in biology, biomedical technology and conservation.

Germplasm cryopreservation includes storage of the sperm, eggs and embryos and contributes directly to animal breeding programmes.

*Author for correspondence: chiahsin@nmmba.gov.tw 
Germplasm cryopreservation also assist the ex situ conservation for preserving the genomes of threatened and endangered species. The establishment of germplasm banks using cryopreservation can contribute to conservation and extant populations in the future. Since the first successful cryopreservation of bull semen (Polge et al. 1949), cryopreserved bull semen has been used to propagate the rare and endangered species using assisted reproduction techniques. Every year, more than 25 million cows are artificially inseminated with frozen-thawed bull semen (Foote 1975) and many bovine calves have been produced using the transfer of cryopreserved embryos into cow (Mapletoft and Hasler 2005). Tissues, cultured cell lines, DNA and serum samples could be frozen and store in cryogene bank. For example, mice and sheep have been generated from frozen-thawed pieces of ovary that have been replaced in a female and stimulated to ovulation. (Gosden et al. 1994; Candy et al. 2000; Sapundzhiev 2008). The principle of testicular cell freezing and transplantation has been demonstrated and is currently used for human male infertility (Clouthier et al. 1996). Significant efforts are being made on non-mammalian species using cryobiology techniques. In fish aquaculture, the successful cryopreservation of gametes and embryos could offer new commercial possibilities, allowing the unlimited production of fry and potentially healthier and better conditioned fish as required. Cryopreservation of reproductive products of many aquatic species has been successfully achieved. Cryopreservation of aquatic sperm is relatively common in the breeding and management of fish species, including salmonid, cyprinids, silurids, and Acipenseridae (família) is well documented (Magyary et al. 1996; Tsvetkova et al. 1996). However, cryopreservation of embryos and oocytes of aquatic species have not been successful, except for eastern oyster eggs (Crassostrea virginica) (Tervit et al. 2005), larvae of eastern oyster (Paniagua-Chavez and Tiersch 2001) and larvae of the sea urchin (Adams et al. 2006).

Cryopreservation technology applied to the preservation of fish gametes in aquaculture plays an important role in seed production, genetic management of broodstock and conservation of aquatic resources. Fish germplasm also plays a significant role in human genomic studies because its relatively small size of the genome makes it easier for sequencing and ideal models for studying the human disease. This would help in identifying the roles for human genes from fish mutations and also in fish models for genes identified by human disease (Brownlie et al. 1998; Barbazuk et al. 2000). Aquatic species preservation would assist the development, protection and distribution of research lines and would offer benefits for restoration of endangered species.

\section{Sperm}

In 1949, Polge et al. (1949) successfully cryopreserved the avian spermatozoa using glycerol as a cryoprotectant. Thereafter, cryopreservation of male gamete became possible. Blaxter (1953) applied a similar approach for fish gametes and reported success with Atlantic herring spermatozoa, achieving approximately $80 \%$ cellular motility after thawing. Since then, cryopreservation of fish sperm has been studied and has been successful in more than 200 species (Kopeika et al. 2007; Tiersch et al. 2007; Tsai et al., 2010) and techniques of sperm management have been established for freshwater and marine fish species, including carp, salmonids, catfish, cichlids, medakas, white-fish, pike, milkfish, grouper, cod, and zebrafish (Scott and Baynes 1980; Harvey and Ashwood-Smith 1982; Stoss and Donaldson 1983; Babiak et al. 1995; Suquet et al. 2000; Van der Straten et al. 2006; Bokor et al. 2007; Tsai et al. 2010). Many studies on cryopreservation of fish sperm have been carried out on economically important freshwater species and attempts to cryopreserve sperm from the marine fish species tended to be more successful when compared with those obtained from the freshwater fish (Tsvetkova et al. 1996). Although freshwater fish sperm are generally more difficult to cryopreserve, the fertilization rates obtained from the cryopreserved marine fish sperm are similar to those obtained with mammalian species (Tsvetkova et al. 1996). Controlled-rate slow cooling in cryopreservation has been mainly used for fish sperm. Common carp has been studied using frozen-thawed sperm with $95 \%$ fertilization and hatching rate.

Salmonid species spermatozoa have been successfully cryopreserved (Lahnsteiner 2000). Another well studied cryopreserved group is cyprinids and some of these cyprinid fishes are widely farmed throughout Asia and Europe. A fertilization and hatching rate of $95 \%$ using the frozen-thawed sperm has been reported for the 
common carp and these results are not significantly different from fresh sperm (Magyary et al. 1996). Tilapias are among the exotic freshwater fishes that have been successfully established for fish farming in Taiwan; they have been cryopreserved successfully and produced 40$80 \%$ motility with cryoprotectant DMSO (Chao et al. 1987). The sperm of more than 30 marine fish species have been cryopreserved successfully (Suquet et al. 2000; Gwo 2000; Van der Straten et al. 2006). Generally, high survival and fertilization capacity has been obtained in frozen-thawed spermatozoa when compared to freshwater species (Drokin 1993; Gwo 2000).

Successful cryopreservation of the sperm of aquatic invertebrate has been carried out for sea urchin, oyster, starfish, abalone and coral (Adams et al. 2004a; Adams et al. 2004b; Gwo et al. 2002; Hagedorn et al. 2006; Kang et al. 2009). Dimethyl sulfoxide has also been reported as a successful cryoprotectant for sperm cryopreservation; the concentration range used was 5 to $30 \%$ for these species. Various levels of motility, ranging from $<5 \%$ to $95 \%$, have been reported for the cryopreserved aquatic invertebrate sperm (Dunn and McLachlan 1973).

\section{Embryos}

Cryopreservation of embryos has become an integral part of assisted reproduction. Successful cryopreservation of embryos is important because the biodiversity of both the paternal and maternal genomes will be preserved. While cryopreservation techniques have been largely established for the mammalian embryos, successful cryopreservation of intact fish embryos has not yet been achieved. Factors limiting fish embryo cryopreservation include their multicompartmental biological systems, high chilling sensitivity, low membrane permeability and their large size, which gives a low surface area to volume ratio (Zhang and Rawson 1995). The effect of such low ratio is a reduction in the rate at which water and cryoprotectants can move into and out of the embryo during cryopreservation (Mazur 1984). Fish embryos are osmoregulators; they are released into the external medium and activated. Then the vitelline envelope separates from the plasma membrane and forms chorion. Studies on the chorion permeability of zebra fish embryos clearly showed that it was permeable to electrolytes and a range of cryoprotectant, including propane-1,2-diol, methanol, DMSO, ethylene (Zhang and Rawson 1996). The chorion structure plays a crucial role as flexible filter for the transport of some materials (Toshimori and Tsuzumi 1976) and protects against the microorganisms (Schoots et al. 1982) Studies on zebra fish embryos have shown that the water permeability of the plasma membrane at different developmental stages remained relatively stable. The permeability to methanol (cryoprotectant) appeared to decrease during embryo development (Zhang and Rawson 1998). This also indicated that there was a gradual reduction in the permeability following the fertilization in zebra fish embryos, as opposed to the generally held belief that the membrane permeability of fish embryos reduced rapidly to minimum shortly after the fertilization (Alderdice 1988).

The studies on the kinetics of subzero chilling injury in Drosophila embryos (Mazur et al. 1992) and chilling sensitivity of zebra fish embryos have demonstrated that chilling injury plays an important role in reduction of embryo survival during the exposure to subzero temperatures (Zhang and Rawson 1995; Hagedorn et al. 1997). Chilling sensitivity has been shown for many species and has been analyzed in fish embryos, including brown trout (Salmo trutta $f$. fario) (Maddock 1974), rainbow trout (Oncorhynchus mykiss) (Haga 1982), carp (Cyprinus carpio) (Dinnyes et al. 1998), fathead minnows (Pimephales promelas) (Cloud et al. 1988), goldfish (Carassius auratus) (Liu et al. 1993) and zebrafish (Danio rerio) (Zhang and Rawson 1995; Zhang et al. 2003). These studies demonstrated that the later stages (after 50\% epiboly) were less sensitive to chilling, but chilling sensitivity increased significantly as the temperature fell below zero. The high chilling sensitivity of fish embryos, especially at early stages, their complex membrane structure and large yolk are the main obstacles to achieve successful cryopreservation of these embryos (Zhang and Rawson 1996). Chilling injury in embryos has been linked to the inhibition of metabolic and enzymatic processes from low temperatures injuries which could be detrimental in the embryonic development such as fish embryos (Dinnyes et al. 1998). Cryoprotectant toxicity follows a similar pattern to chilling sensitivity with later stages being less sensitive to cryoprotectant (Zhang et al. 2005; Zhang et al. 1993; Liu et al. 1993; Suzuki et al. 1995). Several studies have determined membrane permeability for zebra fish embryos (Zhang and Rawson 1998; 
Hagedorn et al. 1997) and membrane permeability to water and most cryoprotectants has been shown to be low (Zhang and Rawson 1996; Zhang and Rawson 1998). Studies on the cryopreservation of zebra fish embryos demonstrated $8 \%$ embryo survival in $2 \mathrm{M}$ methanol at $-25{ }^{\circ} \mathrm{C}$; however, no embryo survival was observed when frozen to -30 ${ }^{\circ} \mathrm{C}$ or below (Zhang et al. 1993).

Cryopreservation studies on the embryos and larvae have been conducted on marine invertebrate such as oysters, sea urchins, polycheate worms, coral and penaeid shrimp species (Liu et al. 2001; Gakhova et al. 1988; Lin et al. 1999; Olive and Wang 1997; Paniagua-Chavez and Tiersch 2001; Hagedorn et al. 2006; Tsai and Lin 2009). However, survivals of most of these species has been inadequate in maintaining the structure and activity of embryos and larvae after freezing to cryogenic temperatures. Embryonic and larval development of marine invertebrates after cryopreservation often showed abnormalities in structure and colour (Odintsova et al. 2001). The problems with invertebrate embryo cryopreservation associated with those identified with the fish embryos are their low membrane permeability and high chilling sensitivity. Although cryopreservation of the embryos has not been fully achieved, considerable progress has been made in understanding the conditions required for fish embryo cryopreservation and this would undoubtedly assist the successful protocol design in the future.

\section{Oocytes}

Oocyte cryopreservation is potentially the best way to preserve the female fertility. Cryopreservation of fish oocyte has been studied (Isayeva et al. 2004; Plachinta et al. 2004; Zhang et al. 2005; Guan et al. 2008; Tsai et al. 2009) which offers several advantages such as the smaller sizes range, much lower water content in oocytes and absence of a fully developed chorion that the permeability to water and solutes in oocyte is higher than embryo. Fish embryos are too large to apply traditional cryopreservation protocol. Immature oocytes can be an alternative for the mature eggs because of their smaller size (Hagedorn et al. 1996). However, there is no practical technique available to induce the small oocyte to mature in vitro. A technique to obtain the mature eggs from the late stage oocytes is available. Thus, the combination of this technique and their cryopreservation could be a breakthrough. However, at present, late stage oocytes cannot be successfully cryopreserved because their size is still not small enough to result in much lower surface area to volume ratio. These reduce the rate at which water and cryoprotectant move into and out of oocytes during the cryopreservation. Developing the methods for cryopreservation of oocytes requires the screening of potential cryoprotectant treatments, evaluation of tolerance to chilling, determination of the appropriate rate of freezing to cryogenic temperatures and rate of thawing. Viability assessment methods of oocytes with trypan blue (TB), fluorescein diacetate (FDA) + propidium iodide (PI) and adenosine triphosphate (ATP) content assay have been developed for quick assessment of viability (Plachinta et al. 2004; Zampolla et al. 2006; Guan et al. 2008; Tsai et al. 2008; Tsai et al. 2009; Tsai et al. 2011; Tsai and Lin 2012). A functional test based on in vitro maturation, followed by germinal vesicle breakdown (GVBD) has also been shown effective for late stage III oocyte (Plachinta et al. 2004).

The permeability of the zebra fish oocyte membrane to water and cryoprotectants has been studied (Zhang et al. 2005) and membrane permeability was shown to decrease with the temperature and permeability was generally lower than those obtained from sea urchin eggs (Adams et al. 2003) but higher than the immature medaka oocyte (Valdez et al. 2005). Studies on zebra fish oocyte chilling sensitivity showed that those oocytes were very sensitive to chilling and their survival decreased with decreasing temperature (Isayeva et al. 2004). Chilling sensitivity in zebra fish oocytes was thought to be due to lipid phase transition of the oocyte membrane (Pearl and Arav 2000). The phase transition in zebra fish oocytes showed that chilling injury could occur when oocytes were exposed to temperatures between 12 to $22^{\circ} \mathrm{C}$ above the water freezing temperatures (Drobnis et al. 1993; Pearl and Arav 2000). Cryopreservation of late stage zebra fish oocytes has been studied using the controlled slow cooling and an optimum cryoprotective medium and cooling rate identified. Guan et al. (2008) showed that although the oocyte viability obtained immediately after freeze-thawing was relatively high with $88 \%$ using TB staining; oocyte viability decreased to $29.5 \%$ after $2 \mathrm{~h}$ incubation at $22{ }^{\circ} \mathrm{C}$. The study also showed that the ATP level in the oocytes decreased significantly after thawing and all oocytes became translucent. Cryopreservation 
of early stage zebra fish oocytes using the controlled slow freezing has been reported by Tsai et al. (2009). The results suggested that $4 \mathrm{M}$ methanol in $\mathrm{KCl}$ buffer was identified as the optimum cryoprotective medium. Although results obtained after the cryopreservation using trypan blue and FDA+PI staining were promising with $69 \%$ and $54 \%$, especially with stage II ovarian follicles, the ADP/ATP ratio assay showed that the energy system of these follicles had been compromised. Apparently the ADP/ATP ratio could be a valuable measure of cellular injury after post-thaw incubation period as it reflected the metabolic and energy status of population as well as indicating some measure of the potential for repair. Furthermore, in vitro culture method is effective for assessing early stage zebra fish oocytes growth competence in vitro. The early stage zebra fish oocytes can be cultured in vitro for $24 \mathrm{~h}$, stage I and II oocytes can grow to the sizes of early stage II and stage III oocytes after hCG treatment. and also can be used for other teleost species (Tsai et al. 2010).

Studies on the cryopreservation of invertebrate oocytes and eggs over the past several decades have been extraordinarily difficult to achieve (Koseoglu et al. 2001; Tsai et al. 2010; Lin et al. 2011; Lin and Tsai 2012). However, it was found that intracellular crystallization occurred in the starfish oocytes at relatively high temperature that was very close to the temperature of extracellular ice formation (Koseoglu et al. 2001). In order to avoid this problem, Hamaratoglu et al. (2005) successfully cryopreserved starfish oocytes using ultra-rapid freezing technique, called vitrification. High chilling sensitivity (Tsai et al. 2009) and low membrane permeability (Guan et al. 2008) of zebra fish oocytes are major obstacles to the development of a successful protocol for their cryopreservation as chilling sensitivity or cold shock can hinder slow cooling processes. Vitrification may be another option to achieve successful cryopreservation for the oocytes.

\section{Blastomeres}

Blastomeres are the cells produced as the result of cell division and cleavage in the fertilized egg. They are totipotent and pluripotent (depending on the stage of embryonic development) having the ability to differentiate into any of the three germ layers or entire organism. They are different from the muscle cells, blood cells or nerves cells. Although cultured somatic-cells from fish have been cryopreserved successfully, their value is limited because of loss of development potential. Cryopreservation of blastomeres can maintain the genetic diversity of both, nuclear genome and mitochondrial DNA (Nilsson and Cloud, 1992). Blastomeres from the early embryos of fish still retain pluripotency (Ho and Kimmel 1993) and their cryopreservation may be a promising approach to preserve the genotypes of zygotes and reconstitution of the organism. Indeed, there are several reports of germ-line chimeras created using the transplantation of blastomeres into goldfish (Yamaha et al. 1997; Kusuda et al. 2004), zebra fish (Lin et al. 1992), medaka (Hong et al. 1998; Wakamatsu et al. 2001) and rainbow trout (Takeuchi et al. 2001) embryos. Kusuda et al. (2004) transplanted the frozen-thaw blastomeres into goldfish embryos and the blastomeres differentiated into primordial germ cells. This report demonstrated that germ-line cells from the cryopreserved blastomeres could develop into mature gametes of chimeric fish because the blastomeres were not damaged by cryopreservation. Therefore, the cryopreservation techniques are very important.

Cryopreservation of blastomeres has been successful in several fish species. In the first reported studies, Harvey (1983) used a two-step freezing procedure, with ice-seeding at $-6^{\circ} \mathrm{C}$, and cooling to $-25^{\circ} \mathrm{C}$, followed by immersion in liquid nitrogen. The survival rate of $84.8 \%$ was obtained after cryopreservation of $50 \%$ epiboly zebra fish blastomeres. However, the results obtained from a very small sample size and freezing rates were not controlled, rather tubes were allowed to equilibrate in the cooled alcohol baths. Lin et al. (2009) demonstrated the effect of cryopreservation on zebra fish blastomeres survival using the controlled slow cooling method. It was shown that DMSO was the most toxic to zebra fish blastomeres. However, DMSO was the best cryoprotectant in terms of survival of zebra fish blastomeres. Therefore, it is possible that the cryoprotective effect of DMSO may be greater than its toxicity effect. Although the survival rate in Lin's results progressed from $25 \%$ (Kopeika et al. 2005) to $70 \%$, it was still lower than that obtained by using two step methods. The comparisons between these studies must take into consideration the different methodology. Vitrification of zebra fish blastomeres was studied more recently and the highest blastomere survival was $93.4 \%$ (Cardona-Costa and Garcia-Ximénez 
2007). Cryopreservation of blastomeres was also carried out in rainbow trout, carp and medaka after post-thawing. Rainbow trout blastomeres have been cryopreserved using the controlled slow freezing procedures with a survival of $95 \%$ (Calvi and Maisse 1998). It has been reported that the controlled slow freezing protocol adopted for rainbow trout was successfully applied to carp blastomeres with survivals of $94 \%$ and $96 \%$ (Calvi and Maisse 1999). Lower survival rates of cryopreserved blastomeres using controlled slow freezing have also been reported for other fish species such as whiting (20\%), medaka (34\%), pejerrey (67\%) and chum salmon (59\%) (Strussmann et al. 1999; Kusuda et al. 2002).

\section{CONCLUSION}

Cryopreservation of gametes and embryos are already routinely applied in the mammalian. Cryopreserved sperm, oocytes and embryos are used for artificial insemination and embryo transfer in the livestock industry. Cryopreservation also has enormous applications in the artificial propagation of widely diverse aquatic organism. Sperm and embryonic cells cryopreservaiton has been successful in a number of teleosts and invertebrate species. However, cryopreservation of embryos and oocytes remain a major challenge. The practical application of cryopreservation in the aquatic species needs more vigorous research efforts in this area and the efforts may be prioritized on endangered, economical value and representative species from various aquatic habitats. Cryopreservation of gametes, embryos and embryonic cells has become of immense value in aquatic biotechnologies which provide an important tool for protecting the endangered species, genetic diversity in aquatic species. The establishment of cryobanks to utilize the cryopreservation worldwide would be a significant and promising task in the future.

\section{REFERENCES}

Adams SL, Hessian PA, Mladenov PV. The potential for cryopreserving larvae of the sea urchin, Evechinus chloroticus. Cryobiology. 2006; 52(1): 139-145.
Adams SL, Hessian PA, Mladenov PV. Cryopreservation of sea urchin (Evechinus chloroticus) sperm. CryoLetters, 2004; 25(4): 287289.

Adams SL, Kleinhans FW, Mladenov PV, Hessian PA. Membrane permeability characteristics and osmotic tolerance limits of sea urchin (Evechinus chloroticus) eggs. Cryobiolog. 2003; 47: 1-13.

Adams SL, Smith JF, Roberts RD, Janke AR, Kaspar HF, Tervit HR, et al. Cryopreservation of sperm of the Pacific oyster (Crassostrea gigas): development of a practical method for commercial spat production. Aquaculture, 2004; 242: 271-282.

Alderdice DF. Osmotic and ionic regulation in teleost eggs and larvae. In: Fish Physiology, editor. W.S. Hoar, D.J. Randall. Academic Press, San Diego, 1988. p. 163-251.

Babiak I, Glogowsky, Brzuska JE, Szumiec J, Adamek $\mathrm{J}$, Cryopreservation of sperm of common carp Cyprinus carpio. Aquaculture Res. 1995; 28: 567571.

Barbazuk WB, Korf I, Kadavi C, Heyen J, Tate S, Wun E, et al. (2000), The syntenic relationship of the zebrafish and human genomes. Genome Res. 2000; 10: 1351-1358.

Blaxter JHS. Sperm storage and cross-fertilization of spring and autumn spawning herring. Nature. 1953; 172: 1189-1190.

Bokor Z, Müller T, Bercsényi M, Horváth L, Urbányi B, Horváth A, Cryopreservation of sperm of two European percid species, the pikeperch (Sander lucioperca) and the Volga pikeperch ( $S$. volgensis). Acta. Biol. Hung. 2007; 58(2): 199-20.

Brownlie A, Donovan A, Pratt SJ, Paw BH, Oates AC, Brugnara $\mathrm{C}$, et al. Positional cloning of the zebrafish sauternes gene: A model for congential sideroblastic anaeia. Nat .Genet, 1998; 20: 244-250.

Calvi SL, Maisse G. Cryopreservation of rainbow trout (Oncorhynchus mykiss) blastomeres: Influence of embryo stage on post-thaw survival rate. Cryobiology. 1998; 36: 255-262.

Calvi SL, Maisse G, Cryopreservation of carp (Cyprinus carpio) blastomeres. Aquat. Living Resour. 1999; 12: 71-74.

Candy CJ, Wood MJ, Whittingham DG, Restoration of a normal reproductive lifespan after grafting of cryopreserved mouse ovaries. Hum. Reprod. 2000; 15(6): 1300-1304.

Cardona-Costa J, García-Ximénez F. Vitrification of zebrafish embryo blastomeres in microvolumes. Vitrification of zebrafish embryo blastomeres in microvolumes. CryoLetters. 2007; 28: 303-309.

Chao NH, Chao WC, Liu KC, Liao IC. The properties of tilapia sperm and its cryopreservation. J. Fish. Biol. 1987; 30: 107-118. 
Cloud JG, Erdahl AL, Graham EF. Survival and continued normal development of fish embryos after incubation at reduced temperatures. Trans. Am. Fish. Soc, 1988; 117: 503-506.

Clouthier DE, Avarbock MR, Maika SD, Hammer RE, Brinster RL. Rat spermatogenesis in mouse testis. Nature. 1996; 381: 418-421.

Dinnyés A, Urbánvi B, Baranvai B, Maqvary I. Chilling sensitivity of carp (Cyprinus carpio) embryos at different developmental stages in the presence or absence of cryoprotectants: work in progress. Theriogenology. 1998; 50(1): 1-13.

Drobnis EZ, Crowe LM, Berger T. Anchordoruy TJ, Overstree tJW, Crow JH. Cold shock damage is due to lipid phase transitions in cell membranes: a demonstration using sperm as a model. J. Exp. Zoo . 1993; 265: 432-437.

Drokin SI. Phospholipid distribution and fatty acid composition of phosphatidylcholine and phosphatidylethanolamine in sperm of some freshwater and marine species of fish. Aquat. Living Resour. 1993; 6: 49-56.

Dunn RS, McLachlan J. Cryopreservation of echinoderm sperm. Can. J. Zool. 1973; 51: 666-669.

Foote RH. (1975) Semen quality from the bull to the freezer: An assessment. Theriogenology. 1975; 3: 219-235.

Gakhova EN, Krasts IV, Naidenko T, Saveleva BI. Embryonic development of the sea urchin after lowtemperature preservation. Ontogenez. 1988; 19: 175180.

Gosden RG, Baird DT, Wade JC, Webb R. Restoration of fertility to oophorectomised sheep by ovarian autografts stored at $-196{ }^{\circ}$ C. Hum. Reprod. 1994; 9: 597-603.

Guan M, Rawson DM, Zhang T. Cryopreservation of zebrafish (Danio rerio) oocytes using improved controlled slow cooling protocols. Cryobiology. 2008; 56: 204-208.

Gwo JC. Cryopreservation of aquatic invertebrate seman: A review. Aquaculture Res. 2000; 31: 259271.

Gwo JC, Chen CW, Cheng HY. Semen cryopreservation of small abalone (Haliotis diversicolor supertexa). Theriogenology. 2002; 58: 1563-1578.

Haga Y. On the subzero temperature preservation of fertilized eggs of rainbow trout. Bull. Jpn. Soc. Sci. Fish. 1982; 48: 1569-1572.

Hagedorn M, Hsu EW, Pilatus U, Wildt DE, Rall WF, Blackband SJ. Magnetic resonance microscopy and spectroscopy reveal kinetics of cryoprotectant permeation in a multicompartmental biological system. Proc. Natl. Acad .Sci., USA 1996; 93(15): 7454-7459.
Hagedorn M, Kleinhans FW, Freitas R, Liu J, Hsu EW, Wildt DE, et al. Water distribution and permeability of zebrafish embryos, (Brachydanio rerio). J. Exp. Zool. 1997; 278: 356-371.

Hagedorn M, Pan R, Cox EF, Hollingsworth L, Krupp D, Lewis TD, Coral larvae conservation: physiology and reproduction. Cryobiology. 1997; 52: 33-47.

Hamaratoglu F, roglu A, Toner M, Sadler KC. Cryopreservation of starfish oocytes. Cryobiolog. 2005; 50: 38-47.

Harvey B. Cooling of embryonic cells, isolated blastoderms and intact embryos of the zebra fish Brachydanio rerio to $-196^{\circ}$ C. Cryobiology. 1983; 20: 440-447.

Harvey B, Ashwood-Smith MJ. Cryopretectant penetration and supercooling in the eggs of samonid fishes. Cryobiology. 1982; 19: 29-40.

Ho RK, Kimmel CB, Commitment of cell fate in the early zebrafish embryo. Science. 1993; 261: 109-111.

Hong Y, Winkler C, Schartl M. Production of medakafish chimeras from a stable embryonic stem cell line. Proc. Natl. Acad. Sci. USA 1998; 95: 36793684.

Isayeva, A, Zhang T, Rawson DM, Studies on chilling sensitivity of zebrafish (Danio rerio) oocytes. Cryobiology. 2004; 49(2): 114-122.

Kang X, Li G, Mu S, Guo M, Ge S. (2009), Acrosome reaction of Chinese mitten-handed crab Eriocheir sinensis (Crustacea: Decapoda) spermatozoa: Promoted by long-term cryopreservation. Aquaculture. 2009; 295: 195-199.

Kopeika E, Kopeika J, Zhang T. Cryopreservation of fish sperm. Methods Mol. Biol. 2007; 368: 203-17.

Kopeika J, Zhang T, Rawson DM, Elgar GS. Effect of cryopreservation on mitochondrial DNA of zebrafish (Danio rerio) blastomere cells. Mutat. Res. 2005; 570: 49-61.

Koseoglu M, Eroglu A, Toner M, Sadler KC. Starfish oocytes form intracellular ice at unusually high temperatures. Cryobiology. 2001; 43: 248-259.

Kusuda S, Teranishi T, Koide N. Cryopreservation of chum salmon blastomeres by the straw method. Cryobiology. 2002; 45: 60-67.

Kusuda S, Teranishi T, Koide N, Nagai T, Arai K, Yamaha E. Pluripotency of cryopreserved blastomeres of the goldfish. J. Exp. Zool, 2004; 301A: 131-138.

Lahnsteiner F. Semen cryopreservation in the Salmonidae and in the northern pike; in special issue: cryopreservation of gametes in aquatic species. Aquacult. Res. 2000; 31: 245-258.

Lin C, Tsai S. The effect of chilling and cryoprotectants on hard coral (Echinopora spp.) oocytes during shortterm low temperature preservation. Theriogenology. 2012; 77: 1257-1261. 
Lin C, Zhang T, Kuo FW, Tsai S. Gorgonian coral (Junceella juncea and Junceella fragilis) oocyte chilling sensitivity in the context of adenosine triphosphate response. Cryoletters. 2011; 32: 141148.

Lin C, Zhang T, Rawson DM. Cryopreservation of zebrafish (Danio rerio) blastomeres by controlled slow cooling. CryoLetter. 2009; 30(2): 132-141.

Lin S, Long W, Chen J, Hopkins N. Production of germ-line chimeras in zebrafish by cell transplants from genetically pigmented to albino embryos. Proc. Natl. Acad. Sci. USA 1992; 89: 4519-4523.

Lin TT, Chao NH, Tung HT. Factors affecting survival of cryopreserved oyster (Crassostrea gigas) embryos. Cryobiology. 1999; 39(2): 192-196.

Liu K, Chou T, Lin H. Cryosurvival of goldfish embryos after subzero freezing. Aquat. Living Resour. 1993; 6: 145-153.

Liu XH, Zhang T, Rawson DM. Differential scanning calorimetry studies of intraembryonic freezing and cryoprotectant penetration in zebrafish (Danio rerio) embryos. J. Exp. Zool. 2001; 290: 299-310.

Maddock BC. A technique to prolong the incubation period of brown trout ova. Progressive Fish Cult. 1974; 36: 219-222.

Magyary I, Urbányi B, Horváth L. Cryopreservation of common carp (Cyprinus carpio L.) sperm II Optimal conditions for fertilization. J. Appl. Ichthyol. 1996; 12: 117-119.

Mapletoft RJ, Hasler JF. Assisted reproductive technologies in cattle: a review. Rev. Sci. Tech. 2005; 24(1): 393-403.

Mazur P. Freezing of living cells: mechanisms and implications. Am. J. Physiol. 1984; 247: C125-C142.

Mazur P, Schneider U, Mahowald AP. Characteristics and kinetics of subzero chilling injury in Drosophila embryos. Cryobiology. 1992; 29: 39-68.

Nilsson EE, Cloud JG. Rainbow trout chimeras produced by injection of blastomeres into recipient blastulae. Proc. Natl .Acad .Sci. USA 1992; 89: 94259428.

Odintsova N, Kiselev K, Sanina N, Kostetsky E. Cryopreservation of primary cell cultures of marine invertebrates. CryoLetters. 2001; 22: 299-310.

Olive PJW, Wang WB. Cryopreservation of Nereis virens (Polychaeta, Annelida) lavae: the mechanism of cryopreservation a different metazoan. Cryobiology. 1997; 34: 284-294.

Palasz AT, Mapletoft RJ. Cryopreservation of mammalian embryos and oocytes: Recent advances. Biotechno. Adv. 1996; 14: 127-149.

Paniagua-Chavez CG, Tiersch TR. Laboratory studies of cryopreservation of sperm and trochophore larvae of the eastern oyster. Cryobiology. 2001; 43(3): 211223.
Pearl M, Arav A. Chilling sensitivity in zebrafish (Brachiodanio rerio) oocytes is related to lipid phase transition. CryoLetters. 2000; 21: 171-178.

Plachinta M, Zhang T, Rawson DM. Studies on cryoprotectant toxicity to zebrafish (Danio rerio) oocytes. CryoLetters. 2004; 25(6): 415-424.

Polge C, Smith AU, Parkes AS. Revival of spermatozoa after vitrification and dehydration at low temperatures. Nature. 1949; 164: 666.

Sapundzhiev E. Conservation of ancient breed small ruminants as frozen embryos. Bulg. J. Vet. Med. 2008; 11: 251-255.

Schoots AFM, Stikkelbroeck JJM, Bekhuis JF, Denuce JM. Hatching in teleost fishes: fine structure changes in the egg envelope during enzymatic breakdown in vivo and in vitro. J. Ultrastruct. Res. 1982; 80: 185196.

Scott AP, Baynes SM. A review of the biology, handing and storage of salmonid spermatozoa. J. Fish Biol. 1980; 17: 707-739.

Stoss J, Donaldson EM. Studies on cryopreservation of eggs from rainbow trout (Salmo gairdneri) and coho salmon (Oncorhynchus Kisutch). Aquaculture. 1983; 31: 51-65.

Strussmann CA, Nakatsugawa H, Takashima F, Hasobe M, Suzuki T, Takai R. Cryopreservation of isolated fish blastomeres: effects of cell stage, cryoprotectant concentration, and cooling rate on post thawing survival. Cryobiology. 1999; 39: 252-261.

Suquet M, Dreanno C, Fauvel C, Cosson J, Billard R. Cryopreservation of sperm in marine fish. Aquaculture Res. 2000; 31(3): 231-243.

Suzuki T, Komada H, Takai R, Arii R, Kozima TT, ( Relation between toxicity of cryoprotectant DMSO and concentration in several fish embryos. Fish Sci. 1995; 61: 193-197.

Takeuchi Y, Yoshizaki G, Takeuchi T. Production of germ-line chimeras in rainbow trout by blastomere transplantation. Mol. Reprod .Dev. 2001; 59: 380389.

Tervit HR, Adams SL, Roberts RD, McGowan LT, Pugh PA, Smith JF, et al. Successful cryopreservation of Pacific oyster (Crassostrea gigas) oocytes. Cryobiology. 2005; 51(2): 142-151.

Tiersch TR, Yang H, Jenkins JA, Dong Q. Sperm cryopreservation in fish and shellfish. Soc. Reprod. Fertil. Suppl. 2007; 65: 493-508.

Toshimori K, Tsuzumi F. The morphology and the function of the oocyte chorion in the teleost, Plecoglossus altivelis. J. Electron Microscopy. 1976; 25: 210.

Tsai S, Lin C. Effects of cryoprotectant on the embryos of banded coral shrimp (Stenopus hispidus), preliminary studies to establish freezing protocols. CryoLetters. 2009; 30(5): 373-381. 
Tsai S, Rawson DM, Zhang T. Development of cryopreservation protocols for early stage zebrafish (Danio rerio) ovarian follicles using controlled slow cooling. Theriogenology. 2009; 71: 1226-1233.

Tsai S, Spikings E, Lin C. Effects of the controlled slow cooling procedure on freezing parameters and ultrastructural morphology of Taiwan shoveljaw carp (Varicorhinus barbatulus) sperm. Aquat. Living Resour. 2010; 23: 119-124.

Tsai S, Spikings E, Huang IC, Lin C. Study on the mitochondrial activity and membrane potential after exposing later stage oocytes of two gorgonian corals (Junceella juncea and Junceella fragilis) to cryoprotectants. CryoLetters, 2011; 32(1): 1-12.

Tsai S, Spikings E, Kuo FW, Lin C. Use of an adenosine triphosphate assay, and simultaneous staining with fluorescein diacetate and propidium iodide, to evaluate the effects of cryoprotectants on hard coral (Echinopora sp.) oocytes. Theriogenology. 2010; 73: 605-611.

Tsai S, Rawson DM, Zhang T. Studies on cryoprotectant toxicity to early stage zebrafish (Danio rerio) ovarian follicle. CryoLetters. 2008; 29(6): 477483.

Tsai S, Rawson DM, Zhang T. Development of in vitro culture method for early stage zebrafish (Danio rerio) ovarian follicles for use in cryopreservation studies.

Theriogenology. 2010; 74: 290-303.

Tsvetkova LI, Cosson J, Linhart O, Billard R. Motility and fertilizing capacity of fresh and frozen-thawed spermatozoa in sturgeons Acipenser baeri and A. ruthenus. J. Appl. Ichthyol. 1996; 12: 107-112.

Valdez MDJr, Miyamoto A, Hana T, Edashige K, Kasai M. Sensitivity of chilling of medaka (Orysias latipes) embryos at various developmental stage. Theriogenology. 2005; 64: 112-122.

Van der Straten KM, Leung LK, Rossini R, Johnston SD. Cryopreservation of spermatozoa of black marlin, Makaira indica (Teleostei: Istiophoridae). CryoLetters. 2006; 27(4): 203-209.
Wakamatsu Y, Pristyaznhyuk I, Ju B, Niwa K, Ladygina T, Kinoshit M, et al. Fertile and diploid nuclear transplants derived from embryonic cells of a small laboratory fish, medaka (Oryzias Latipes). Proc. Natl. Acad. Sci. USA 2001; 98: 1071-1076.

Yamaha E, Mizuno T, Hasebe Y, Yamazaki F. Chimeric fish produced by exchanging upper parts of blastoderms in goldfish blastulae. Fish. Sci. 1997; 63: 514-519.

Zampolla T, Rawson DM, Zhang T. Development of new viability assessment methods for zebrafish (Danio rerio) oocytes. Cryobiology. 2006; 58:16.

Zhang T, Rawson DM. Studies on chilling sensitivity of zebrafish (Brachydanio rerio) embryos. Cryobiology. 1995; 32: 239-246.

Zhang T, Rawson DM. Feasibility studies on vitrification of zebrafish (Brachydanio rerio) embryos. Cryobiology. 1996; 33: 1-13.

Zhang T, Isayeva A, Adams SL, Rawson DM. Studies on membrane permeability of zebrafish (Danio rerio) oocytes in the presence of different cryoprotectants. Cryobiology. 2005; 50: 285-293.

Zhang T, Liu XH, Rawson DM. Effects of methanol and developmental arrest on chilling injury in zebrafish (Danio rerio) embryos. Theriogenology. 2003; 59(7): 1545-1556.

Zhang T, Rawson DM, Morris GJ. Cryopreservation of pre-hatch embryos of zebrafish (Brachydanio rerio). Aquat. Living Resour. 1993; 6: 145-153.

Zhang TT, Rawson DM. Permeability of dechorionated 1-cell and 6-somite stage zebrafish (Brachydanio rerio) embryos to water and methanol. Cryobiology. 1998; 37: 13-21. 


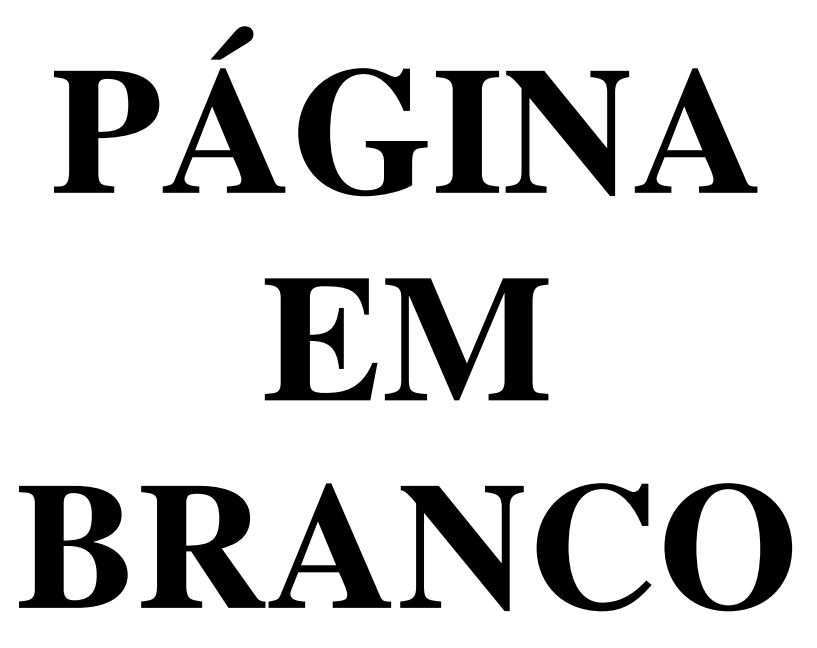

